\title{
Aktives Fehlermanagement durch eine teamorientierte Arbeitskultur
}

Beat Brändle, Daniel Scheidegger

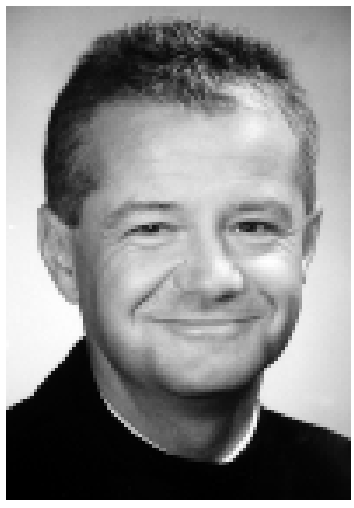

Beat Brändle, Captain A320, Swiss International Air Lines

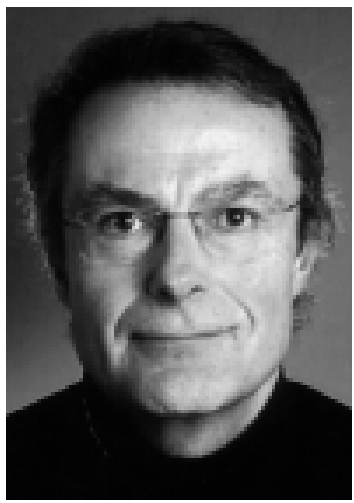

Prof. Daniel Scheidegger, Chefarzt Dep. Anästhesie, Universitätsspital Basel
Korrespondenz:

Dr. med. Christoph Harms

Hirslanden

Klinik Aarau

Schänisweg

CH-5001 Aarau

harms.consulting@besonet.ch www.hadmedical.ch

\section{«Rhode Island surgeon operates on wrong side of the head!»}

Dies war die Schlagzeile einer amerikanischen Lokalzeitung am 15. Dezember 2004. Beim Lesen des Artikels glaubt man, dass der Chirurg den Unterschied von links und rechts nicht kennt. In Wirklichkeit handelte es sich um einen bekannten Neurochirurgen, der an jenem Morgen drei Patienten mit einem Meningeom operieren sollte. Bei den ersten zwei war der Befund links, beim dritten rechts. Aus organisatorischen Gründen wurde am Operationstag die Reihenfolge geändert. Wie sich im nachhinein herausstellte war zwar das ganze übrige Team im betreffenden Operationssaal informiert, nicht aber der verantwortliche Chirurg. Da dann noch zusätzlich die CT-Bilder seitenverkehrt aufgehängt wurden, wurde der 2. Patient falsch gelagert, abgedeckt und die Schädeldecke auf der falschen Seite aufgemacht. Verurteilt wurde der Chirurg, weil die jetzt üblichen Verfahren, um «wrong side surgery» zu vermeiden (Markierung, Time-out usw.), nicht angewendet wurden. Eine einzelne Person wurde verurteilt, obwohl das ganze Team versagt hat!

Wie in vielen anderen Bereichen, arbeiten auch in der Medizin die Menschen in einem komplexen und hochtechnologischen Umfeld. Teamleistungen stehen im Vordergrund, da der einzelne, aufgrund des hohen Spezialisierungsgrades und des optimierten Prozessablaufs, gar nicht mehr in der Lage ist, eine Leistung alleine zu erbringen. Da gleichzeitig bei gesteigerter Produktion die Kosten gesenkt werden müssen, wird das menschliche Verhalten im Team ausschlaggebend für die Sicherheit im System. Bei über $70 \%$ aller medizinischen Zwischen- und Unfälle ist der Mensch zumindest als Mitverursacher beteiligt. Niemand kann diese Tatsache ad acta legen, ohne selber Mitverantwortung für mögliche Fehlleistungen zu tragen.

In der Luftfahrt wurden vor über 20 Jahren, aufgrund einiger fataler Unfälle, aus diesen Erkenntnissen die Konsequenzen gezogen, und es setzte sich die Einsicht durch, dass die Konsequenzen von Fehlern der einzelnen Mitarbeiter durch eine teamorientierte Arbeitskultur und eine verbesserte Kommunikation minimiert werden können. Auch der eingangs beschriebene medizinische Zwischenfall hätte durch eine solche teamorientierte Arbeitskultur vermieden werden können.

Dabei hängen die Sicherheit und Effizienz eines Teams nicht nur von den individuellen Fertigkeiten und Arbeitseinstellungen der einzel-

\section{Gestion active de fautes \\ par une culture axée \\ sur le travail en équipe}

«Rhode Island surgeon operates on wrong side of the head!» Le 15 décembre 2004, ce fait divers faisait la une d'un journal local américain. Attribuer des fautes et distribuer des sanctions après des incidents médicaux n'aide ni les personnes concernées ni l'institution mais encourage un climat de méfiance réciproque et une dissimulation des fautes dont les conséquences sont finalement supportées par le patient. Les médecins accomplissent leur travail dans un environnement complexe et de haute technologie. Au bout du compte, ce sont les prestations réalisées en équipe qui prévalent, chaque spécialiste fournissant sa contribution dans un processus complexe. Dans ce système, le comportement humain au sein de l'équipe est déterminant pour la sécurité. L'homme est impliqué dans plus de $70 \%$ des incidents et accidents médicaux! Forts de ce constat, les domaines industriels à haut risque ont déclaré obligatoire la formation de leurs collaborateurs en travail d'équipe. La firme HADmedical tente pour la première fois d'appliquer ces idées à la médecine de manière conséquente et offre depuis I'an 2000 une formation en équipe interdisciplinaire dans le domaine médical en englobant toute la hiérarchie. Bien que ce projet soit sur le bon chemin, force est de constater que tout changement de culture est un travail de longue haleine! 
Während der Schlussphase eines überaus ehrgeizigen und prestigeträchtigen Projektes vermeldet eine Projektgruppe Probleme in ihrem Bereich, welche momentan unlösbar seien und konsequenterweise zum Abbruch der Arbeiten oder mindestens zu einer Terminverschiebung führen müssten. Kurz vor der Lancierung wurde schriftlich festgehalten: Wir können das Problem nicht lösen - das ist eine rote Warnflagge! Das Projekt wurde weitergeführt, obwohl dem Management der offensichtliche Mangel bewusst war, und es endete in einer Katastrophe. Eine ganze Nation war erschüttert; Space Shuttle Challenger explodiert 1986 wenige Sekunden nach dem Start wegen eines fehlerhaften Dichtungsringes. nen Menschen ab, sondern ganz entscheidend vom Verhalten der Teammitglieder zueinander. Faktoren die in diesem Zusammenhang eine entscheidende Rolle spielen sind:

- eine offene und konstruktive Kommunikationskultur;

- ein ziel- und lösungsorientiertes Gruppenklima;

- ein konstruktiver Umgang mit Konflikten, der sachorientierte Lösungen ermöglicht.

Von zentraler Bedeutung in diesem Zusammenhang ist das Bewusstsein, dass wir alle, unabhängig von unserer Position und Ausbildung - als Menschen -, Fehler machen. Nun gibt es verschiedene Möglichkeiten, mit solchen Fehlleistungen umzugehen. Man kann sie ignorieren, versuchen, sie zu vertuschen, oder macht andere Menschen und/oder Umstände dafür verantwortlich. Solches Verhalten kennt jedoch nur Verlierer, in der Medizin meist auf der Seite der Patienten.

Ein ganz anderer Ansatz ist der Gedanke, dass wir aus Fehlern lernen können, um diese so in Zukunft zu vermeiden oder zumindest einschränken zu können.

Ein aktives Fehlermanagement erhöht die Sicherheit für den Patienten, wirkt sich positiv auf die Arbeitseffizienz, die Zufriedenheit des Patienten und nicht zuletzt auf die Mitarbeiterzufriedenheit aus. Die Qualität der gesamten Dienstleistung steigt.

\section{Was heisst aktives Fehlermanagement?}

Gemachte Fehler sollen sachlich und ohne Vorurteile und Vorverurteilungen offengelegt und mit den Betroffenen besprochen und analysiert werden. Das wiederum ist nur möglich, wenn eine Kultur des Vertrauens innerhalb der entsprechenden Organisationseinheit herrscht. Jede einzelne Person muss wissen, dass sie aufgrund eines Fehlers nicht einfach bestraft oder schikaniert wird, sondern dass ein konstruktiver Lösungsansatz gesucht wird, um denselben Fehler in Zukunft zu vermeiden. Dies heisst nicht, dass bei grobfahrlässigem Verhalten keine entsprechenden Konsequenzen durch den/die Verantwortlichen getragen werden müssen, aber es geht darum, dass dieselben Fehler nicht ein zweites Mal gemacht werden.

\section{Umsetzung}

In den neunziger Jahren eigneten sich viele Fluggesellschaften, unter anderem auch die Swissair, grosses Know-how in der Schulung ihrer Mitarbeiter/innen in teamorientiertem Verhalten an. Zuerst war dies nur auf das Cockpit beschränkt, wurde aber später auf die gesamte Flugzeugbesatzung ausgedehnt.

Da dieser Themenbereich Neuland war, wurden verschiedene Wege ausprobiert, diese Schulungen im Arbeitsalltag zu implementieren und die neuen Vorgaben umzusetzen. Es galt, sowohl die weltweit unterschiedlichen nationalen und betrieblichen, kulturellen Bedürfnisse zu berücksichtigen als auch die Anliegen und Fertigkeiten der betroffenen Mitarbeiter/innen aufzunehmen und zu integrieren.

In einem eigentlichen «trial and error»-Verfahren stellte sich schliesslich heraus, dass solche Weiterbildungen folgende drei Grundsätze berücksichtigen müssen, damit sie zum geforderten Ziel, der Verbesserung und Erhöhung der Sicherheit, führen:

- Die Schulung muss verhaltensorientiert sein: Alle Teammitglieder müssen sich für ihr persönliches Verhalten verantwortlich fühlen, um eine kooperative Arbeitsatmosphäre aktiv mitzuschaffen.

- Die Schulung muss interdisziplinär und hierarchieübergreifend sein: Jedes Teammitglied hat seinen individuellen Wirkungskreis. Wenn ein Team als ganzes versteht, dass die Leistung jedes einzelnen zur Gesamtleistung einen Beitrag bildet, erwächst daraus auch die Erkenntnis, dass jedes Teammitglied an der Qualität des Endproduktes Verantwortung mitträgt.

- Die Schulung soll einen ganzheitlichen Aspekt zeigen: Die praktische Umsetzung von theoretischem Wissen im Bereich Verhalten ist von zentraler Bedeutung. Damit wird gewährleistet, dass das neu erworbene Wissen schnell und prozessoptimierend im täglichen Arbeitsumfeld umgesetzt werden kann.

Am Universitätsspital Basel wurde dieser Ansatz Ende der 90er Jahre erstmalig aufgenommen und versucht, konsequent auf die Medizin zu übertragen - HADmedical $=$ Human Aspects Development in der Medizin*. In Zusammenarbeit mit Spezialisten aus der Luft- und Raumfahrt (NASA) kunft 3 - bis 4 mal pro Jahr einen kleinen Beitrag aus den erwähnten Themenbereichen vorstellen. 
wurden die Erfahrungen aus diesem Bereich auf das medizinische Umfeld umgesetzt und der Versuch gestartet, eine interdisziplinäre Teamschulung aufzubauen. Das Projekt hat sich in der Zwischenzeit erfreulich entwickelt. Aus der systematischen Auswertung der Teilnehmerrückmeldungen konnten unter anderem folgende Erkenntnisse gewonnen werden:

- Der konkrete Nutzen aus einer teamorientierten Schulung wird für die Organisation erst wahrgenommen, wenn die Mehrheit eines Teams/einer Organisation einen solchen Kurs besucht hat.

- Fehler in der Medizin waren bis vor kurzem noch ein Tabuthema. Aus einer Klinik, welche einen Grossteil ihrer Pflegenden in die Kurse geschickt hat, wurde berichtet, dass auf der Bettenabteilung ein deutlicher «Kulturwandel» hin zu einer offeneren Kommunikation im Gange sei. Über Fehler könne nun in einer ganz anderen, nicht anklagenden Weise gesprochen, entsprechende Lehren gezogen und Verbesserungsmassnahmen ohne Bestrafung eingeleitet werden.

- Kulturwandel ist ein langwieriger, kontinuierlicher und unspektakulärer Prozess, der einen langen Atem verlangt (wurde beispielsweise in der Swissair vor gut 20 Jahren mit kleinen
Schritten eingeführt und geht heute bei der Swiss immer noch weiter).

- Die Sicherheitskultur darf nicht nur ein Thema für die Mitarbeiter/innen an der Front sein, sondern muss es auf allen Stufen der gesamten Organisation sein. Angefangen bei der Personalrekrutierung, der Aus- und Weiterbildung bis hin zu Refresherkursen und Reportingsystemen in der Qualitätssicherung und im Controlling. Dabei schliesst es sämtliche Hierarchiestufen ein und muss letztlich von allen aktiv gelebt werden. Der Führung kommt hierbei eine besondere Vorbildfunktion zu!

- Fehlermeldesysteme können erst in ihrem eigentlichen Sinn genützt werden, wenn eine entsprechende Kulturveränderung stattgefunden hat.

In diesem Sinne sind die HADmedical-Kurse einerseits einmal «Eyeopener», bieten aber anderseits zweifellos Anstoss und Motivation für eine nachhaltige Änderung der Fehlerverarbeitung in unserem Gesundheitswesen. Ob aus der Saat eine Frucht wird, ist nicht nur von ihrer Pflege abhängig, sondern auch vom Boden, auf den sie fällt. 